\title{
A See-through Holographic Head-mounted Display with The Large View Angle
}

\author{
Zhidong Chen, Xinzhu sang, * Qiaojun Lin, Jin Li, Xunbo Yu, Xin Gao, Binbin Yan, Kuiru \\ Wang,ChongxiuYu, Songlin XIE
}

\begin{abstract}
A novel solution for the large view angle holographic head-mounted display (HHMD) is presented. Divergent light is used for the hologram illumination to construct a large size three-dimensional object outside the display in a short distance. A designed project-type lens with large numerical aperture projects the object constructed by the hologram to its real location. The presented solution can realize a compact HHMD system with a large field of view. The basic principle and the structure of the system are described. An augmented reality (AR) prototype with the size of 50 $\mathrm{mm} \times 40 \mathrm{~mm}$ and the view angle above 60 degrees is demonstrated.
\end{abstract}

Keywords: Holography; Head-mounted display; Field of view

\section{Introduction}

Near-eye display is the key technology in both virtual reality (VR) and augmented reality (AR) head-mounted display (HMD) systems, which utilizes optical solutions to enable the human eye focus on display devices placed near to eyes. Optical elements are usually used to refract the light from the display devices as if it was coming in from a distance source [1][2]. However, these approaches make the human eye observe a plane of image floating in the air and only the depth cue of binocular disparity is provided, which results in the mismatch of convergence and accommodation for three-dimensional (3D) display. Although the light field display [3][4] can support nearly correct convergence, accommodation, binocular disparity and retinal defocus depth cues, the spatial resolution is considerably reduced. A lightweight super multi-view display is required to achieve a large number of volume pixels, which faces many difficulties in industry fabrication [5]. Holographic display is an alternative solution that can reconstruct the wavefront of three dimensional objects. It can satisfy all human physiological requirements, which can find potential applications in the display technique on HMD [6][7]. Some attempts were done to improve the practical performance of HHMD in the aspects of full-color display [8], computer-generated hologram (CGH) acceleration [9][10], and the field of view (FOV) broadening [11]. Fourier transform optical system (FTOS) is the most commonly used FOV broaden method, which increases the configuration length, making a bulky HHMD system. Although a simple FTOS adopting only one lens is introduced [11], it confronted the restriction of numerical aperture to build a wider FOV HHMD system. At the state of the art, a HHMD system with a FOV of above 20 degrees has not been reported yet. Here, a novel and simple HHMD method is presented, which can realize a large FOV of more than 60 degrees.

\section{Principle}

The configuration of the proposed method is shown in Fig 1. The size of the proposed prototype is practically suitable for mobile devices applications. It consists of one spatial light modulator (SLM), one point source, one polarizer, one compound lens and one polarization filter (PF). A linearly polarized divergent light from a point source through a polarizer is served as the reference wave for the hologram illumination. A compound lens with a large numerical aperture is placed between the SLM and the 
observing eye. As the holographic display is a reflective-type SLM in the system, a beam splitter is inserted between the SLM and the compound lens to offer an off-axis illumination, preventing the point source and the polarizer from blocking the diffracted light. The observing eye is located at the conjugate plane of the point source about the lens. In order to synchronously observe real and virtual images, a half mirror is used as a combiner that combines the environmental light and the virtual light together.

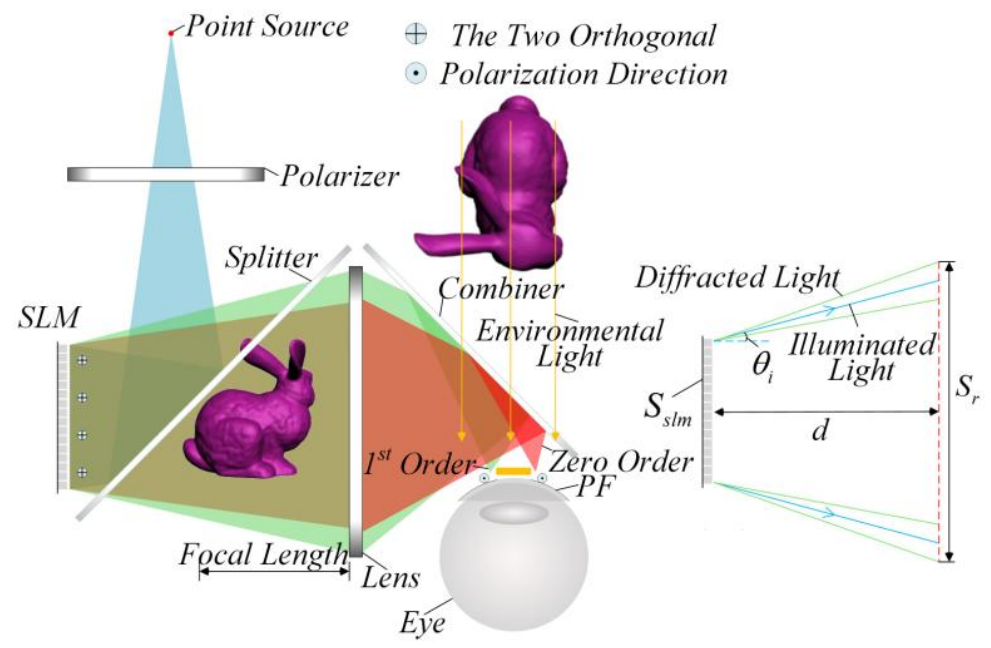

Fig. 1 The diagram of the HHMD system

The proposed HHMD optical system is aspired by the current commercial VR helmets (eg. Occulus Rift). The HMD is enable to achieve a FOV exceed 90 degrees due to its large screen size. The VR helmets project images on a screen to the distance of distinct vision, whereas our system projects a three dimensional object constructed by the hologram to its real position. In electro-holography, the construction of the object wave relies on the diffraction of the pixels of the hologram. The maximum size of the reconstructed object $S_{r}$ from the hologram depends on the divergence angle of the illuminated light $\theta_{i}$, the size $S_{s l m}$ and the pixel pitch $p$ of the SLM, and the reconstructed distance $d$ from the SLM is

$$
S_{r} \approx\left|S_{s l m} \pm 2 d \cdot \tan \theta_{i}\right|+\frac{\lambda d}{p}
$$

where $\lambda$ is the wavelength of the reference light and the sign \pm depends on the property of the reference that is positive for the divergent light and negative for the convergent light. For our proposed approach, a divergent light is used as the reference light to construct a larger object for projection outside the hologram display in a short distance, realizing a wide view angle.

In the HHMD system, the lens has three functions, which is shown in Figure 2. The first one is broadening the FOV. As shown in Figure 2(a), without the lens, most of the diffracted wave generated by the vast majority pixels of the SLM could not enter the pupil of the observing eye, and the FOV is just the limitation of the diffraction angle of the SLM. The lens can converge the diffracted wave over a common area in a short distance. With the lens, a view window (VW) is generated in the image plane of the point source. As the SLM has periodic openings, it can create equidistantly staggered diffraction orders in the viewing plane. Because the light intensity decreases towards higher diffraction orders, the position between the zero-th order and the first order is chosen as the VW. The observing eye views holographic images through the VW. The distance of the VW from the lens $d_{3}$ is given following the Gaussian imaging formula, 
where $d_{1}$ is the distance of the point source from the SLM, $d_{2}$ is the distance between the SLM and the lens, and $f$ is the focal length of the lens. The size of VW $S_{w w}$ also can be derived as

$$
S_{v w}=\frac{\lambda d_{1}}{p} \cdot \frac{d_{3}}{d_{1}+d_{2}}
$$
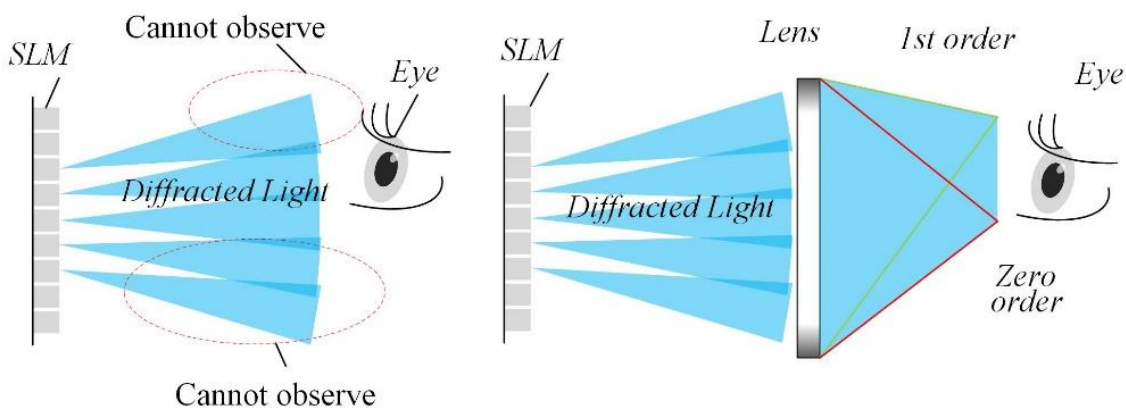

(a)

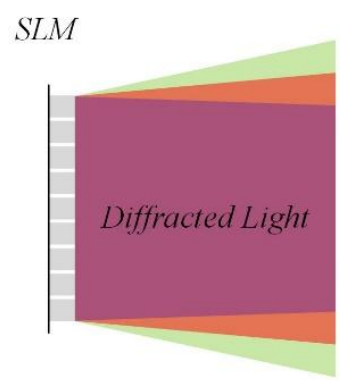

Aliasing!

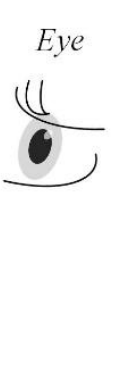

Eye SIM

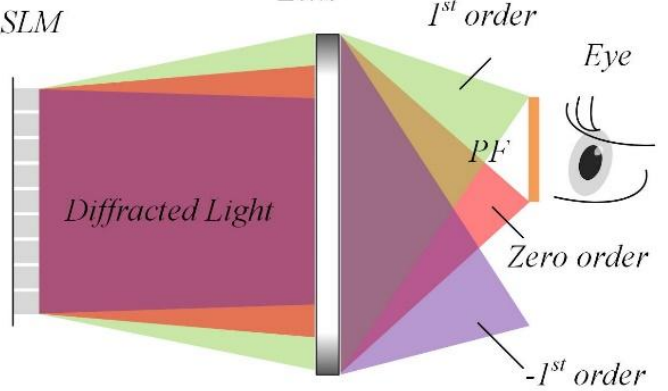

(b)

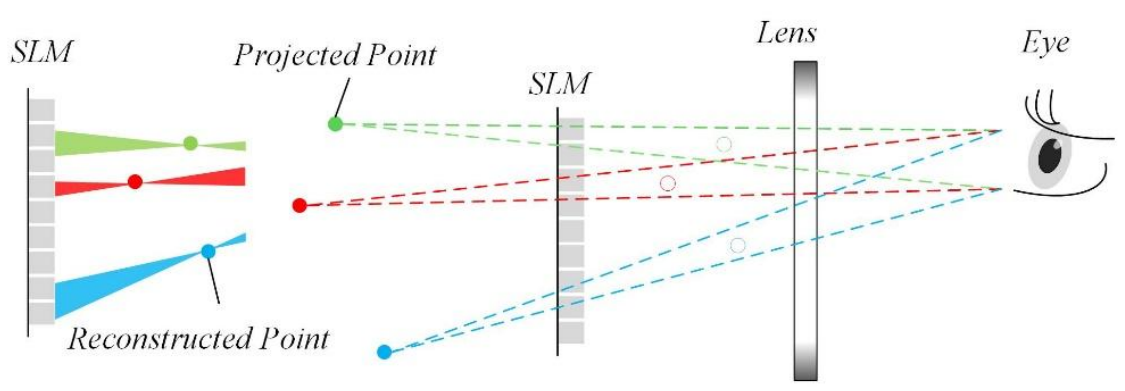

(c)

Fig.2 Functions of the lens

However, as the pixel pitch of the current commercial SLM $p$ is not small enough, the pupil size of normal human eyes (average from $2.5 \mathrm{~mm}$ to $5.0 \mathrm{~mm}$ ) exceeds the size of VW, and the observing eye will receive more than one orders. The diffraction orders will overlap on the retina of the eye if no any auxiliary technique is used. To address this problem, a PF is used. The PF can be integrated into a contact lens for the 
observing eye. The center path of the PF is a small aperture that enables the target wave in the VW to pass through the pupil of the eye. The outer region contains a polarizer, and the polarization direction is orthogonal to the light diffracted from the SLM. With this design, other interferential orders can be prevented from bypassing the pupil of the eye. The environmental light consists of unpolarized broadband light, so that half of the light is able to pass through the PF, enabling the viewer to see the environment with normal vision. The second function of the lens as shown in Figure 2(b) is to remove the unwanted lights easily with the PF, as the unwanted lights are converged at the image plane of the lens. Without the lens, different order of the diffracted light will be mixed together, and aliasing appears in the observed hologram. The final function of the lens as shown in Figure 2(c) is to project the constructed object with the hologram to the target spatial position that it should be located. With this method, the tangent value of the half view angle is equal to the ratio of the exit pupil radius of the system with the exit relief.

\section{Optical design}

From the above analysis, the display effect of the HHMD system largely relies on the design of the lens. The lens design principle is to make the configuration display system compact and the FOV large within the allowed range of the aberrations, which means that the focal length of the lens cannot be too long and the aperture should be large enough. Figure 3 shows the side view of proposed HHMD with optimized optical design. Two convex lenses are assembled, forming a compound lens of a large numerical aperture. The upper left of Figure 3 is the independent design of the core lenses. The exit pupil with a diameter of $4 \mathrm{~mm}$ is where the eye is placed to view the reconstructed 3D object. The optimized maximum half view angle is 32 degrees and the effective focal length is $24 \mathrm{~mm}$. The bottom left of Figure 3 is the structure design of the HHMD system. A diffracted grating with a same fringe period as the pixel space of SLM is used to simulate the diffraction performance of the SLM. In the diagram, the lines with green color are the zero order and the blue is the first order of the diffracted waves. The right of Figure 3 plots the modulation transfer functions (MTFs) of 5 fields within 91 grid points. The MTFs of the most fields are above 0.1 at $25 \mathrm{lp} / \mathrm{mm}$.
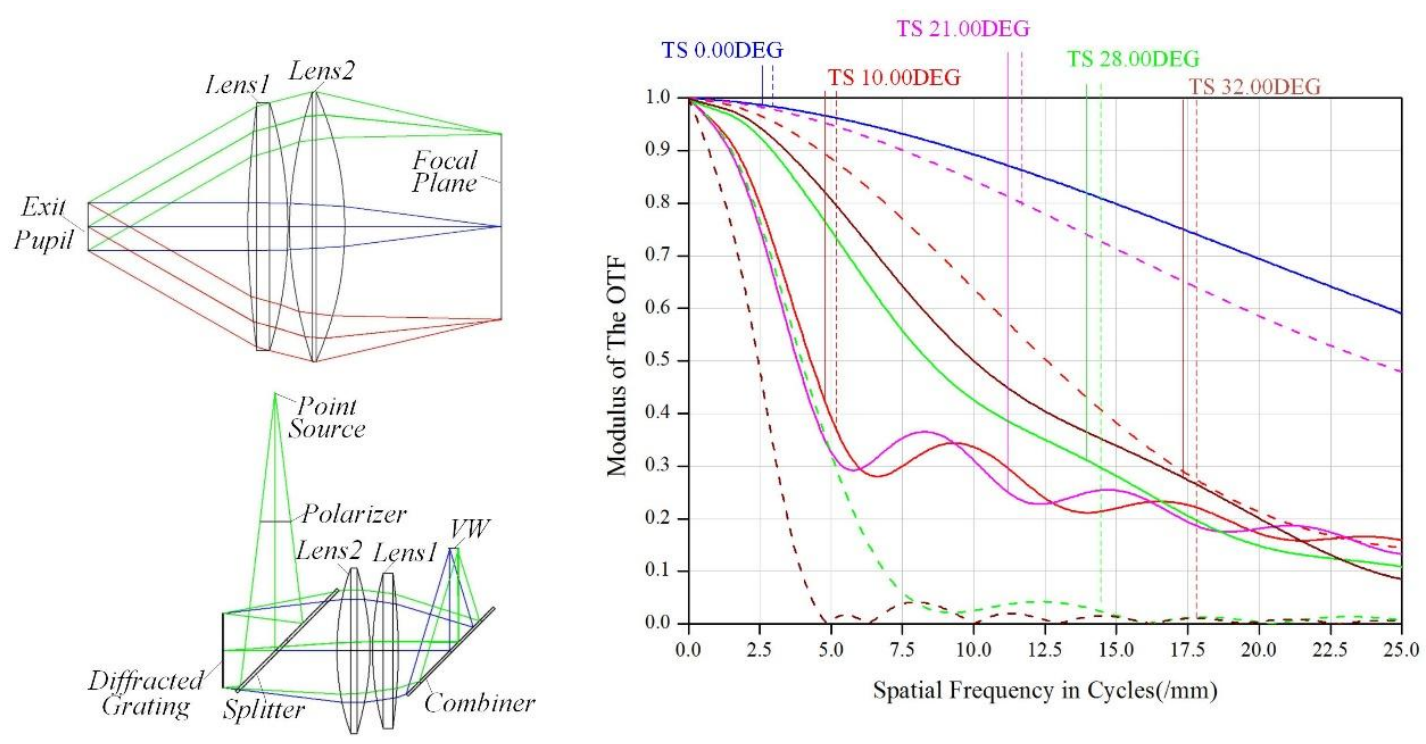

Fig.3 Optical design of HHMD system and the MTF plots of the designed lens

\section{CGH generated algorithm}

There are several methods to calculate the computer generated hologram (CGH), and a point-based method is used to generate the hologram. Point-based methods regard the $3 \mathrm{D}$ object to be recorded as clouds of 
independent light points, with each object point propagating spherical wave. The complex amplitude distribution $C_{m}(u, v)$ on the hologram plane is as follows,

$$
C_{m}(u, v)=\sum_{n=1}^{N} \frac{A_{n}}{R_{m n}} \exp \left(i \frac{2 \pi}{\lambda} R_{m n}\right)
$$

where $(u, v)$ is the coordinate on the $\mathrm{CGH}, A_{n}$ is the intensity of a point source indexed by $\mathrm{n}$, and $\mathrm{N}$ is the total number of 3D object points. $R_{m n}=\sqrt{\left(x_{n}-u_{m}\right)^{2}+\left(y_{n}-v_{m}\right)^{2}+d_{n}^{2}}$ is the distance between a 3D object point with coordinate $\left(x_{n}, y_{n}, d_{n}\right)$ and a position with the coordinate $\left(u_{m}, v_{m}\right)$ on hologram plane. As the third function of the lens described above, the propagation position from each object point to $\operatorname{hologram}\left(x_{n}, y_{n}, d_{n}\right)$ is not the actual observed position. The relationship between the propagation position and the actual observed position of each object point should follow the imaging characteristics of the lens.

\section{Experiment and Results}

A HHMD system is set up based on the structure diagram as shown in Fig.1. A tiny red color LED serves as the point light source. A polarizer modulates the polarization direction of the light scatted from the SLM to be orthogonal to the polarization direction of the PF.

The optical parameters of the system are shown in Table. 1. The structure of the system is compact. A photography of the constructed experimental system is shown in Fig. 4(a) and the dimension of the system is also indicated. Fig. 4(b) shows the reconstructed numbers " 1 " and " 2 " captured by a camera separately without the PF and with the PF. The photographs are captured using a Point Grey Flea3 camera with a 0-2.0 mm variable aperture lens supporting a minmum f-number of 2.0. It refers that the PF can effectively block the interferential light. Fig. 4(c) is an AR scene to illustrate the FOV of the HHMD system. A board and a physical tape are located at $40 \mathrm{~cm}$ away from the camera. The tape on the board shows the effective size of the reconstructed rabbit is about $48 \mathrm{~cm}$, so that a FOV is $2 \tan ^{-1}(48 / 2 / 40)=61.9^{\circ}$, which confirms the theoretic prediction. To the best of our knowledge, it is the largest FOV HHMD system.

$4(\mathrm{~d})$ is the photographs of two reconstructed letters in different depth. When the letter "A" is focused by the
camera, the letter " $\mathrm{B}$ " is blurred. On the other hand, when the letter "B" is in focus, the letter " $\mathrm{A}$ " gets
blurred. It shows that this system can support correct accommodation effect.

Table 1 Experimental parameters

\begin{tabular}{|c|c|}
\hline \multicolumn{2}{|c|}{ SLM } \\
\hline Pixel pitch & $8.0 \mathrm{um}$ \\
\hline Resolution & $1920 \times 1080$ \\
\hline Refresh rate & $60 \mathrm{~Hz}$ \\
\hline Wavelength of LED \\
\hline Red & $625 \mathrm{~nm}$ \\
\hline \multicolumn{2}{|c|}{ Lens 1 } \\
\hline Focal length & $36 \mathrm{~mm}$ \\
\hline Aperture & $35 \mathrm{~mm} \times 19 \mathrm{~mm}$ \\
\hline \multicolumn{2}{|c|}{ Lens2 } \\
\hline Focal length & $56 \mathrm{~mm}$ \\
\hline Aperture & $36 \mathrm{~mm} \times 21 \mathrm{~mm}$ \\
\hline \multicolumn{2}{|c|}{ Others } \\
\hline
\end{tabular}




\begin{tabular}{|c|l|}
\hline Diameter of PF & $1.0 \mathrm{~mm}$ \\
\hline FOV & $62 \mathrm{deg}$. \\
\hline
\end{tabular}

In summary, a large FOV see-through type HHMD system is demonstrated. The size of the system is small so that it is suitable for mobile devices. A PF is used as a contact lens in this system and it is located near the aperture of the camera. As additional vision correction methods can be integrated into the contact lens, other glasses are not required for the observer, which can improve the utility and comfort of HHMD [12].

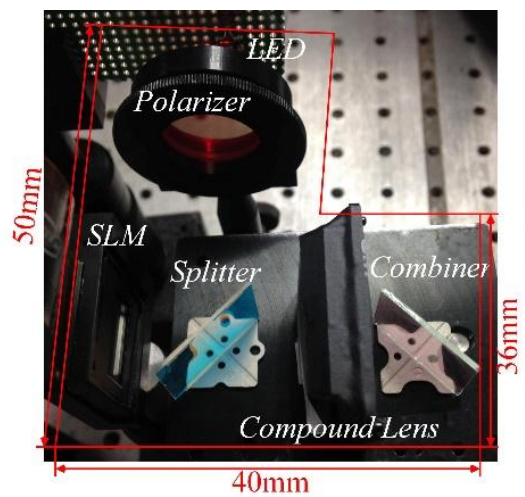

(a)

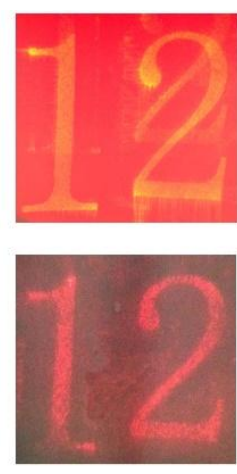

(b)

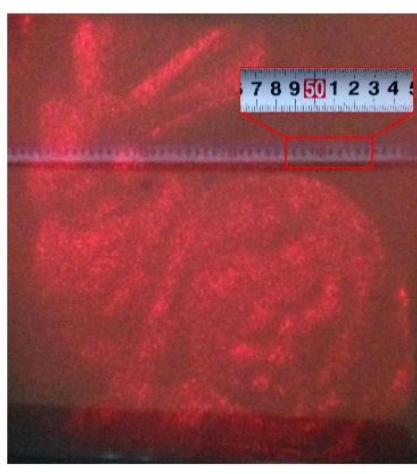

(c)
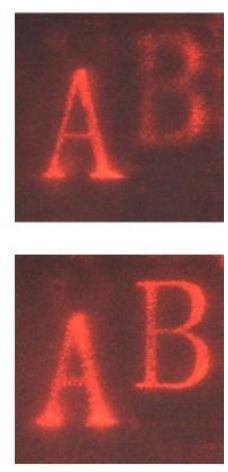

(d)

Fig.4(a) The photograp of the HHMD system (b) The reconstructed number taken by a camera. Upper:

without PF. Bottom: with PF (c) An AR scene perceived through the HHMD system (d) Two reconstructed

$$
\text { letters in different depth }
$$

\section{Funding sources and acknowledgments.}

This work is partly supported by the "863" Program (2015AA015902), the National Science Foundation of China (61575025), the fund of the State Key Laboratory of Information Photonics and Optical Communications, and the Program of Beijing Science and Technology Plan (D121100004812001).

\section{References}

1. O. Cakmakci and J. Rolland, Head-worn displays: a review. J.DISP.TECHNOL. 2, 3 (2006), p. 199-216.

2. J. Yang, W. Liu, W. Lv, D. Zhang, F. He, Z. Wei and Y. Kang, Method of achieving a wide field-of-view head-mounted display with small distortion. Opt. Lett. 38,12 (2013), p. 2035-2037.

3. D. Lanman and D. Luebke, Near-eye light field displays. ACM.T.GRAPHIC. 32, 6 (2013), p. 220

4. H. Hua and B. Javidi, A 3D integral imaging optical see-through head-mounted display. OPT.EXPRESS. 22 , 11 (2014), p. 13484-13491

5. Y. Takaki, Super multi-view display with 128 viewpoints and viewpoint formation. IS\&SPIE Electronic Imaging (2009).

6. T. Takemori, 3-dimentional display using liquid crystal devices - fast computation of hologram. HODIC Circular. 17, 3 (1997)

7. H.E. Kim, N. Kim, H. Song, H.S. Lee and J.H. Park, Three-dimensional holographic display using active shutter for head mounted display application. IS\&SPIE Electronic Imaging (2011).

8. E. Moon, M.Kim, J. Roh, H. Kim and J. Hahn, Holographic head-mounted display with RGB light emitting diode light source. OPT.EXPRESS. 22, 6 (2014), p. 6526-6534

9. J.S. Chen and D.P. Chu, Improved layer-based method for rapid hologram generation and real-time interactive holographic display applications. OPT.EXPRESS. 23, 14 (2015), p. 18143-18155 
10. Z. Chen, X. Sang, Q. Lin, J. Li, X. Yu, X. Gao, B. Yan, C. Yu, W. Dou and L. Xiao, Acceleration for computer-generated hologram in head-mounted display with effective diffraction area recording method for eyes. COL. 14,8 (2016)

11. T. Yoneyama, T. Ichikawa, Y. Sakamoto, Semi-portable full-color electro-holographic display with small size. SPIE OPTO (2014)

12. J. Legerton and Randall Sprague, Methods and apparatus for constructing a contact lens with optics. US 8142016 B2 (2012) 